Research Paper

\title{
Apparent Diffusion Coefficient Quantification in Determining the Histological Diagnosis of Malignant Liver Lesions
}

\author{
Konstantinos Drevelegas ${ }^{1}$, Katerina Nikiforaki ${ }^{2}$, Manolis Constantinides ${ }^{1}$, Nickolas Papanikolaou ${ }^{2}$, \\ Lavrentios Papalavrentios ${ }^{3}$, Ioanna Stoikou ${ }^{5}$, Paul Zarogoulidis ${ }^{6}{ }^{\varpi}$, Georgia Pitsiou ${ }^{6}$, Athanasia Pataka ${ }^{6}$, \\ John Organtzis ${ }^{6}$, Eleni Papadaki ${ }^{6}, K^{\prime}$ Konstantinos Porpodis ${ }^{6}$, Ioanna Kougioumtzi ${ }^{7}$, Ioannis Kioumis ${ }^{6}$, \\ Constantinos Kouskouras ${ }^{4}$, Evaggelos Akriviadis ${ }^{3}$, Antonios Drevelegas ${ }^{1}$
}

1. Department of Radiology, Interbalkan Medical Centre, Thessaloniki, Greece;

2. Research \& Development, N.Papanikolaou \& Associates LLC, Crete, Greece;

3. Department of Internal Medicine-Gastroenterology, Interbalkan Medical Centre, Thessaloniki, Greece;

4. Department of Radiology, Ahepa University Hospital, Thessaloniki, Greece;

5. Department of Microbiolgy, George Gennimatas Hospital, Thessaloniki, Greece

6. Pulmonary Department-Oncology Unit, “G. Papanikolaou” General Hospital, Aristotle University of Thessaloniki, Thessaloniki, Greece;

7. Surgery Department (NHS), University General Hospital of Alexandroupolis, Alexandroupolis, Greece.

$\square$ Corresponding author: Paul Zarogoulidis, M.D, PhD. Pulmonary Department-Oncology Unit, "G. Papanikolaou" General Hospital, Aristotle University of Thessaloniki, Thessaloniki, Greece. Fax: 00302310992424 Mobile: 00306977271974 E-mail: pzarog@hotmail.com.

๑ Ivyspring International Publisher. Reproduction is permitted for personal, noncommercial use, provided that the article is in whole, unmodified, and properly cited. See http://ivyspring.com/terms for terms and conditions.

Received: 2015.10.21; Accepted: 2016.01.22; Published: 2016.03.29

\begin{abstract}
Purpose: Diffusion Weighted Imaging is an established diagnostic tool for accurate differential diagnosis between benign and malignant liver lesions. The aim of our study was to evaluate the role of Histogram Analysis of ADC quantification in determining the histological diagnosis as well as the grade of malignant liver tumours. To our knowledge, there is no study evaluating the role of Histogram Analysis of ADC quantification in determining the histological diagnosis as well as the grade of malignant liver tumours.

Methods: During five years, 115 patients with known liver lesions underwent Diffusion Weighted Imaging in 3Tesla MR scanner prior to core needle biopsy. Histogram analyses of ADC in regions of interest were drawn and were correlated with biopsy histological diagnosis and grading.

Results: Histogram analysis of ADC values shows that 5th and 30th percentile parameters have statistically significant potency of discrimination between primary and secondary lesions groups ( $P$ values 0.0036 and 0.0125 respectively). Skewness of the histogram can help discriminate between good and poor differentiated ( $p$ value 0.17 ). Discrimination between primary malignancy site in metastases failed for the present number of patients in each subgroup.

Conclusion: Statistical parameters reflecting the shape of the left side of the ADC histogram can be useful for discriminating between primary and secondary lesions and also between well differentiated versus moderate or poor. For the secondary malignancies, they failed to predict the original site of tumour.
\end{abstract}

Key words: Diffusion Weighted Imaging, histogram analysis, liver lesions, biopsy.

\section{Introduction}

Primary liver cancer is the sixth leading cancer globally [1], while liver metastases are predominant with a ratio of 40:1 [2]. MRI has proven to be a useful modality in diagnosis and in time-to-depth re-examinations due to the very good contrast-to-noise ratio, variety of available contrast mechanisms and its non-ionizing radiation use. Diffusion weighted imaging is an established modality which provides diagnostic information that can be related to tissue microarchitecture through the 
amount of restriction of water mobility predominantly within the extracellular space. A quantitative biomarker than can be derived from DWI is called Apparent Diffusion Coefficient (ADC) and has been shown to help in accurate differential diagnosis between benign and malignant focal liver lesions [1].

Despite the fact that radiological modalities have been improved and scientific research has been put towards that end, uncertainty in the differential diagnosis remains, leaving biopsy as the gold standard of diagnosis. The aim of this study was to evaluate the ability of ADC quantification to determine the histological diagnosis as well as the grade of malignant liver tumors.

\section{Materials and methods}

\section{Patients}

During a 5 year period (March 2010 - March 2015) 115 patients with focal liver lesions underwent core needle biopsy under CT guidance. Prior to the biopsy, ADC values of focal lesions were measured in 3T MR scanner (General Electric, HDxT). Thirty eight patients were excluded from the study because either the lesions were less than $5 \mathrm{~mm}$ in diameter, or because the DWI images were severely compromised due to a. ghosting artifacts (ascites or no proper patient cooperation in breathing), b. low SNR. Hence, retrospective analysis was performed for the 77 patients (age range $24-84$, mean age 62, male $n=45$, female $n=32$ ) for whom no exclusion criterion was met. The distribution of lesions with respect to the liver segments was: segment II $n=2$, segment IV $n=13$, segment $\mathrm{V} n=18$, segment VI $n=11$, segment VII $n=12$, segment VIII $n=21$.: Primary lesions: HCC $n=15$, Cholangiocarcinoma $n=17$; The distribution of the metastatic lesions primary site is: breast $n=12$, colorectal $n=10$, lung $n=8$, pancreatic $n=11$, prostate $\mathrm{n}=4$, melanoma $\mathrm{n}=4$, ovarian $\mathrm{n}=1$, stomach $\mathrm{n}=1$. Multiple lesions were found in 42 of the patients. Mean lesion size was equal to $32 \mathrm{~mm}$.

\section{MR Imaging}

All MRI images were acquired with a 3 Tesla imager (Signa HDxT; GE Medical Systems, Milwaukee, WI) with twinspeed gradient system providing up to $50 \mathrm{mT} / \mathrm{m}$ of gradient amplitude). All images were obtained with an 8-channel torso phased array coil.

Echo-planar Diffusion weighted Imaging was performed utilizing respiratory triggering, prior to intravenous administration of contrast. A Single Shot Echo Planar Imaging diffusion sequence (DW-EPI) was acquired in combination with Parallel imaging with Array Spatial Sensitivity Encoding Technique
(ASSET) factor of 2 to minimize geometrical distortions (3). The TR was 10,000 ms, TE ranged between 67 and $69 \mathrm{~ms}$, slice thickness $6 \mathrm{~mm}$, gap between slices $1 \mathrm{~mm}$, matrix $128 \times 128$, field of view $400 \mathrm{~mm}$, number of excitations 4 and receiver Bandwidth $1953 \mathrm{~Hz}$. The total acquisition time ranged between 4 and 5 minutes due to the variation of respiratory patterns between the patients. Two b-values were used, 0 and $1000 \mathrm{~s} / \mathrm{mm}^{2}$. ADC quantification was done by means of the ADC Map Plugin on Osirix.

Following the calculation of ADC values on a pixel by pixel basis, histogram analysis was performed utilizing an in-house developed software tool (DrEye Histogram Plugin, ICS FORTH, Heraklion, Greece). Apart from ADC maps, both b0 and b1000 maps were used to perform histogram analysis. Multiple regions of interest constituting a volume of interest (VOI) were manually drawn on b1000 images on the basis of high signal intensity and then copied to the ADC maps. Areas of necrosis as identified on b0 were excluded from the VOIs. Pixels assigned to the lesion were segmented by a "magic wand" tool on the basis of high signal on b1000 with adjustable user-depended degree of tolerance and then copied to the ADC map by DrEye Histogram Plugin. ADC histograms were generated from all pixels belonging to the VOI, and the following parameters were calculated for each patient: min, max, mean, median, standard deviation, skewness, kurtosis, and 5th, 30th, 70th, and 95th percentiles.

In order to dissociate the software metrics from lesion size, area under the histogram was normalized to unity for all patients. Statistical analysis was carried out using the software package SPSS. The above histogram parameters were compared for the two patient groups (primary or secondary), as well as for subgroups according to histopathologing grading or primary cancer origin for the latter group. P-values less than 0.05 were considered statistically significant.

\section{Biopsy}

Prior to the beginning of the biopsy all patients completed the consent form and were consecutively sedated. Subsequently, all patients underwent core needle biopsy under CT guidance with guns 16 or 18 gauge. The obtained specimens were preserved in formalin and sent to the Department of Pathology for histological examination.

\section{Histology}

Biopsy tissue was fixed in 10\% Neutral Buffered Formalin (NBF) and immediately transferred to pathology. After processing tissue is embedded in paraffin and blocks created. Each block was sectioned 
at 3 microns and two $H \& E$ and 6-10 unstained slides were created for ancillary techniques. After reading of the slides and evaluation of immunohistochemical (IHC) (where necessary) diagnosis was rendered and in case of tumors classification and grading were appointed. All processes were accredited through ISO 15189.

\section{Results}

Among the 115 patients, malignancies were found in 77 of them, out of which primary malignacies were 27 , while secondary were 50 . Tables 1 and 2 show different types of lesions and their corresponding mean ADCs.

As shown in Tables 1 and 2, there is frequently substantial overlap between ADC values of HCC and metastases, as well as among metastatic lesions coming from different primary malignancies. Subsequently the determination of a cut off value based on mean ADC or standard deviation was not attempted since a symmetric distribution cannot be assumed. A histogram based statistical evaluation can elucidate more statistical features about the distribution of ADC values within certain percentage intervals of the histogram (percentiles), about the influence of the outliers, or about the skewness and kurtosis of the distribution.

Table 1. ADC correlation with histopathological findings

\begin{tabular}{|c|c|c|c|c|}
\hline & & $\begin{array}{l}\text { Min ADC } \\
\left(X 10^{-3}\right) \\
\mathrm{mm}^{2} / \mathrm{s}\end{array}$ & $\begin{array}{l}\operatorname{Max} \\
\operatorname{ADC}\left(X 10^{-3}\right) \\
\mathrm{mm}^{2} / \mathrm{s}\end{array}$ & $\begin{array}{l}\text { Mean } \\
\operatorname{ADC}\left(X_{10}-3\right) \\
\mathrm{mm}^{2} / \mathrm{s}\end{array}$ \\
\hline \multirow[t]{2}{*}{$\begin{array}{l}\text { Primary } \\
(n=27)\end{array}$} & $\begin{array}{l}\text { Hepatocellular Carcinoma } \\
(\mathrm{n}=15)\end{array}$ & 0,001 & 3.502 & 1,159 \\
\hline & Cholangiocarcinoma $(n=12)$ & 0,001 & 4.303 & 1,258 \\
\hline \multirow{8}{*}{$\begin{array}{l}\text { Secondary } \\
(n=50)\end{array}$} & breast $(n=12)$ & 0.051 & 3.747 & 1,117 \\
\hline & colorectal $(n=10)$ & 0.031 & 5.349 & 1,296 \\
\hline & lung $(n=8)$ & 0.005 & 4.06 & 1,055 \\
\hline & pancreatic $(n=10)$ & 0.011 & 2.889 & 1.069861 \\
\hline & prostate $(n=4)$ & 0.014 & 2.718 & 1.019816 \\
\hline & melanoma $(n=4)$ & 0.01 & 3.896 & 0,951 \\
\hline & ovarian $(n=1)$ & 0,472 & 2,276 & 0,991 \\
\hline & stomach $(n=1)$ & 0,208 & 1,687 & 0,954 \\
\hline
\end{tabular}

A two fold analysis was performed, investigating statistical discriminants between primary (Group A), and metastatic lesions (Group B), and also between degree of histopathological tissue differentiation; well differentiated lesions comprised group 1 (6 patients), moderate differentiation group 2 (30 patients) and poor differentiation constituted group 3 (29 patients), indifferently of primary or secondary nature of malignancy. Intermediate levels of differentiation were assigned to the lower level.

Table 2: ADC values correlation with differentiation findings

\begin{tabular}{|c|c|c|c|}
\hline & & Differentiation & $\begin{array}{l}\text { mean ADC } \\
\left(\mathrm{X} 10^{-3}\right) \mathrm{mm}^{2} / \mathrm{s}\end{array}$ \\
\hline \multirow[t]{8}{*}{ Primary $(n=27)$} & \multirow{4}{*}{$\begin{array}{l}\text { Hepatocellular } \\
\text { Carcinoma }(n=15)\end{array}$} & poor $(\mathrm{n}=4)$ & 1.006 \\
\hline & & moderate $(n=7)$ & 1.222 \\
\hline & & well $(n=3)$ & 1,238 \\
\hline & & $\begin{array}{l}\text { no differentiation } \\
(\mathrm{n}=1)\end{array}$ & 1,318 \\
\hline & \multirow{4}{*}{$\begin{array}{l}\text { Cholangiocarcinoma } \\
(\mathrm{n}=12)\end{array}$} & poor(n=5) & 1,284 \\
\hline & & moderate $(\mathrm{n}=5)$ & 1,427 \\
\hline & & well( $(n=1)$ & 1,127 \\
\hline & & $\begin{array}{l}\text { no differentiation } \\
(\mathrm{n}=1)\end{array}$ & 1,414 \\
\hline \multirow{24}{*}{$\begin{array}{l}\text { Secondary } \\
(n=50)\end{array}$} & \multirow[t]{4}{*}{ breast $(n=12)$} & poor $(n=5)$ & 1,084 \\
\hline & & moderate $(n=5)$ & 1,240 \\
\hline & & well(n=1) & 0,949 \\
\hline & & no diff $(\mathrm{n}=1)$ & 0,843 \\
\hline & \multirow[t]{3}{*}{ colorectal $(\mathrm{n}=10)$} & poor $(n=2)$ & 1,266 \\
\hline & & $\begin{array}{l}\text { moderate }(n=5) \\
\text { well }(n=0)\end{array}$ & 1,333 \\
\hline & & no diff $(n=1)$ & 1,260 \\
\hline & \multirow[t]{2}{*}{ lung $(n=8)$} & poor $(\mathrm{n}=6)$ & 1,147 \\
\hline & & $\begin{array}{l}\text { moderate }(n=2) \\
\text { well }(n=0)\end{array}$ & 0,782 \\
\hline & \multirow[t]{4}{*}{ pancreatic $(n=10)$} & poor $(n=5)$ & 1,095 \\
\hline & & moderate $(\mathrm{n}=1)$ & 1.153 \\
\hline & & well $(n=1)$ & 1,013 \\
\hline & & no diff $(n=1)$ & 1,026 \\
\hline & \multirow[t]{4}{*}{ prostate $(n=4)$} & poor $(n=1)$ & 0.898 \\
\hline & & moderate $(n=0)$ & \\
\hline & & well $(n=0)$ & \\
\hline & & no diff $(n=3)$ & 1.061 \\
\hline & \multirow[t]{3}{*}{ melanoma $(n=4)$} & poor $(n=1)$ & 1,220 \\
\hline & & $\begin{array}{l}\text { moderate }(n=0) \\
\text { well }(n=0)\end{array}$ & \\
\hline & & no diff $(n=3)$ & 0.860 \\
\hline & \multirow[t]{2}{*}{ ovarian $(n=1)$} & poor $(n=0)$ & \\
\hline & & $\begin{array}{l}\text { moderate }(n=1) \\
\text { well }(n=0)\end{array}$ & 0,990 \\
\hline & \multirow[t]{2}{*}{ stomach $(\mathrm{n}=1)$} & poor $(n=0)$ & \\
\hline & & $\begin{array}{l}\text { moderate }(\mathrm{n}=1) \\
\text { well }(\mathrm{n}=0)\end{array}$ & 0,954 \\
\hline
\end{tabular}

In 27 patients with primary liver malignancy the mean ADC value was $\left(1,24 \pm 0.16 \times 10^{-3} \mathrm{~mm}^{2} / \mathrm{s}\right.$, liver cholangiocarcinoma $1.34 \pm 0.27 \times 10^{-3} \mathrm{~mm}^{2} / \mathrm{s}$ ), while 51 patients with secondary liver malignancy (group B), the mean $\mathrm{ADC}$ value was $1.11 \pm 0.295 \times 10^{-3} \mathrm{~mm}^{2} / \mathrm{s}$. Among 16 measured histogram metrics, analysis of variance (ANOVA) highlights 5 parameters that have statistically significant potency of discrimination between the two groups (mean, median, harmonic mean, $5^{\text {th }}$ percentile, $30^{\text {th }}$ percentile) as presented in Table 3. Among those the $5^{\text {th }}$ percentile exhibits the lower $p$ value of 0.0036 , with the second being the $30^{\text {th }}$ percentile with a corresponding $p$ value of 0.0125 .

The second question attempted by this study was to investigate the discriminant power of the same 
16 metrics in order to differentiate between degrees of cell differentiation. Skewness exhibited a $\mathrm{p}$ value below 0.05 for differentiation between group 1 (mean skewness 0.54, SD 0.66) and 3 (mean skewness 1.24, SD 0.34) groups ( $p$ value 0.048 ) and also between group 2 (mean skewness 0.60, SD 0.51 ) and group 3 (p value of 0.17 ) but failed to differentiate between group 1 and 2 (Table 4). All other 15 ADC histogram metrics of liver focal lesions were not significant.

Discrimination between primary malignancy sites in metastases failed for the present number of patients in each subgroup.
Table 3: Comparison between primary and metastatic liver tumours. Statistical parameters derived from histogram analysis with $p$ value $<0.05$.

\begin{tabular}{llll}
\hline $\begin{array}{l}\text { Apparent Diffusion } \\
\text { Coefficient metrics } \\
\left(\mathrm{mm}^{2} / \mathrm{s}\right)\end{array}$ & Primary $(\mathrm{N}=27)$ & Metastatic $(\mathrm{N}=51)$ & p value \\
\hline Mean ADC & $1.240(0.242)$ & $1.107(0.295)$ & 0.0491 \\
Median ADC & $1.219(0.246)$ & $1.077(0.288)$ & 0.0340 \\
Harmonic Mean & $1.158(0.238)$ & $0.987(0.321)$ & 0.0180 \\
5th percentile & $0.837(0.189)$ & $0.667(0.259)$ & 0.0036 \\
30th percentile & $0.109(0.198)$ & $0.937(0.268)$ & 0.0125 \\
\hline
\end{tabular}

Table 4: Comparison of Mean Skewness between groups (degree of differentiation).

\begin{tabular}{|c|c|c|c|}
\hline Group & $\mathbf{N}$ & Skewness & $\begin{array}{l}\text { Standard Deviation } \\
\text { (SD) }\end{array}$ \\
\hline 1 (well) & 6 & 1.24 & 0.34 \\
\hline 2 (moderate) & 29 & 0.60 & 0.51 \\
\hline 3 (poor) & 30 & 0.53 & 0.66 \\
\hline Group pair & $1-2$ & $1-3$ & $2-3$ \\
\hline$p$ value & 1.000 & 0.048 & 0.171 \\
\hline
\end{tabular}
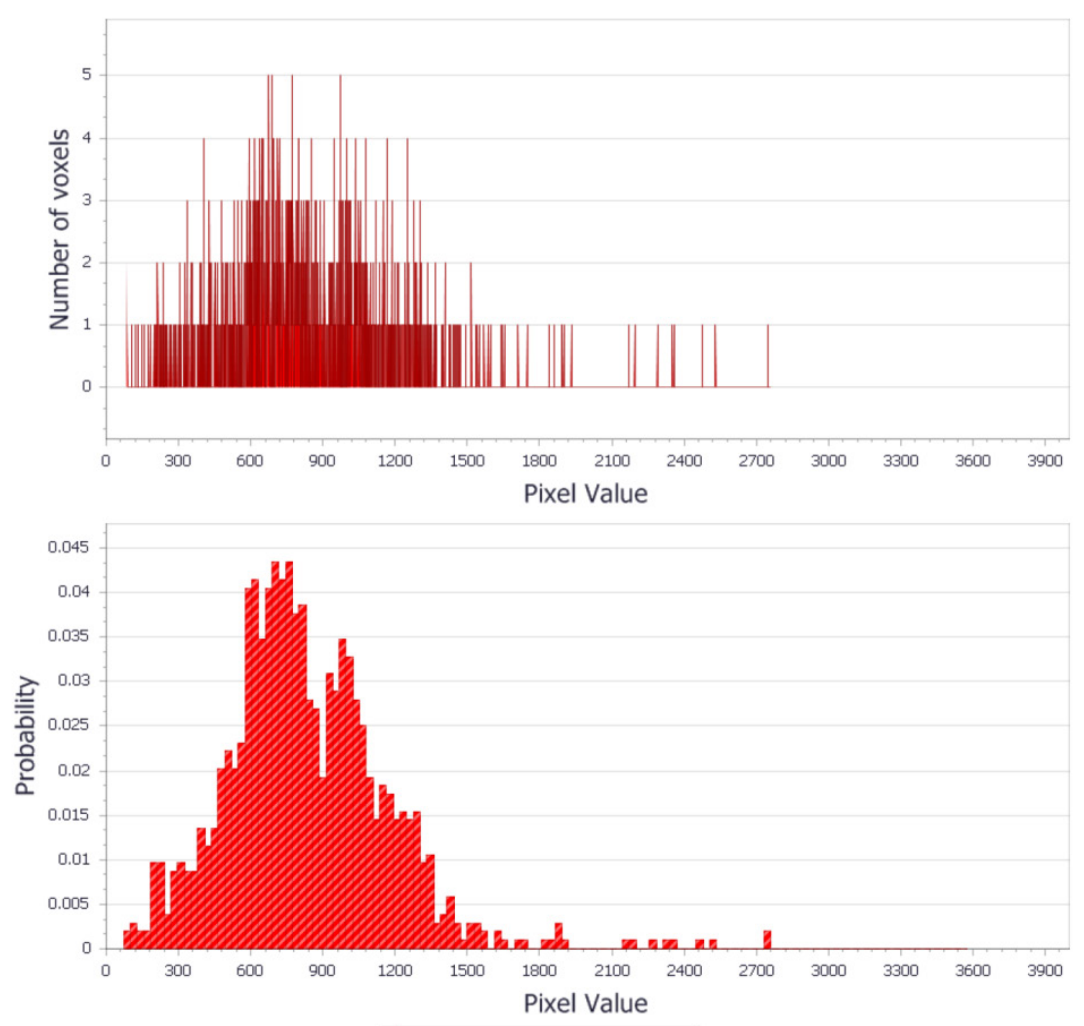

$\boldsymbol{\Delta}$ lesion-1040 Points $\mathbb{Z}$ lesion (pdf)

\section{Statistics for lesion}

$\begin{array}{lll}\text { Min: } 86 & \text { Kyrtosis: } 6.205 & \text { Percentile } 5 \%: 339 \\ \text { Max: } 2750 & \text { Skewness: } 0.986 & \text { Percentile 30\%: } 668 \\ \text { Mean: } 842.381 & \text { Peak Position: } 675 & \text { Percentile } 70 \%: 995 \\ \text { Median: } 800.000 & \text { Peak Height: } 5 & \text { Percentile } 95 \%: 1360\end{array}$

Statistics for lesion (pdf)

$\begin{array}{lll}\text { Min: } 86 & \text { Kyrtosis: } 6.827 & \text { Percentile } 5 \%: 334 \\ \text { Max: } 2746 & \text { Skewness: } 1.101 & \text { Percentile 30\%: } 646 \\ \text { Mean: } 830.751 & \text { Peak Position: } 702 & \text { Percentile 70\%: } 982 \\ \text { Median: } 786.000 & \text { Peak Height: } 0.043 & \text { Percentile } 95 \%: 1346\end{array}$

Figure 1: sample output from the DrEye histogram analysis tool. 


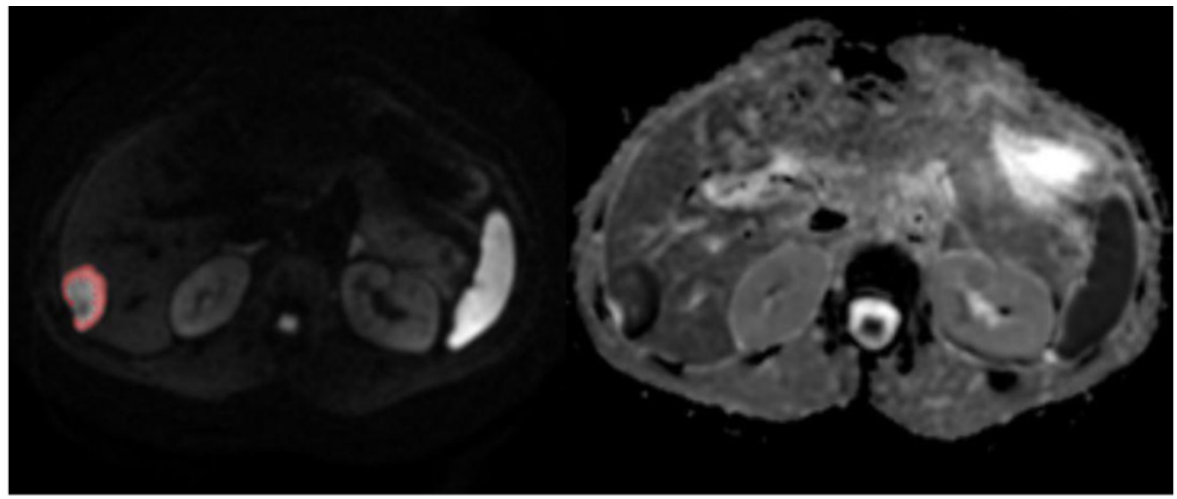

Figure 2 : DrEye Lesion segmentation on b1000 and normalized ADC histogram.

\section{Discussion}

Two of the frequently encountered challenges of diffusion weighted imaging in the body are the limited SNR and the contamination of the image by respiratory related ghost artefacts. Parallel imaging techniques have been utilized (ASSET, acceleration factor 2), in order to minimize acquisition time and echo time was kept to the minimum possible value in order to mitigate high signal ghosting in the phase encoding direction. In the current study, the fact that imaging was performed in a 3 Tesla scanner resulted in adequate SNR, despite the use of parallel imaging and moreover permitted the choice of an appropriate high $b$ value to increase lesion conspiquity. A value of $1000 \mathrm{~s} / \mathrm{mm}^{2}$ was chosen, thus facilitating lesion detection, and also increasing ADC value reproducibility as compared to lower b values [4]. Low tissue SNR can be a source of systematic ADC underestimation and can be a potential source of error in ADC value determination [6,7]. The number of non-zero $b$ values was kept to 1 (only b 1000) because of software limitations.

Several studies attempt lesion characterization from the mean ADC value for a certain type of pathology [6]. From the current study results, it was obvious that the most useful information from the dispersion of ADC values is in the lower end of the interval, as the most powerful discriminants between primary and secondary liver malignancies were the $5^{\text {th }}$ and $30^{\text {th }}$ percentile with the median value $\left(50^{\text {th }}\right.$ percentile) immediately after. The frequently used metric of the mean is slightly below the frequently used $p$ threshold of 0.05 (0.0491), thus proving the need for more accurate metrics. It is also obvious that the useful information that the histogram conveys is in the low ADC values and can be disregarded when averaged over a very large interval in order to specify a certain cut off value, especially when taking into account the heterogeneity of certain lesions containing different signal patterns on ADC maps [5].
Furthermore, such an analysis based on individual percentiles can minimize the effect of outliers, especially from pixels at the boundaries of the lesion exhibiting higher ADC values or from noise.

The fact that the most accurate metric for discrimination between different degrees of cell differentiation is histogram skewness can be attributed to the asymmetric shape of ADC value distribution having mean value different from the median.

All groups exhibit positive skew, meaning that the left end of the histogram (low ADC values) is more densely populated as intuitively expected for malignant lesions. Between the three different groups, group 1 (well differentiated) has the highest value of skewness (1.24 versus 0.60 and 0.53 for group 2 and 3 respectively) giving away a larger difference between the median and mean value and a more abrupt left side of the histogram for well differentiated lesions than moderate or poor.

\section{Conclusion}

The current study combined high SNR data from a $3 \mathrm{~T}$ scanner with histogram based analysis to evaluate whether ADC metrics can discriminate primary from secondary liver malignancies, degree of cell differentiation, and original site of tumour. Statistical parameters reflecting the shape of the left side of the ADC histogram can be useful for the first two inquiries, while failed for the latter probably because of inadequate sample size. The initial assessment with the method described is very important for the patient. The patient can be informed quickly for his disease and additional information can be used by the treating physician in order to proceed with his diagnostic and treating algorithm.

\section{Conflict of Interest}

None to declare. 


\section{References}

1. World Health Organization. World Cancer Report 2014. 2014: Chapter 1.1. ISBN 9283204298

2. Pickren JW, Tsukada Y, Lane WW. Liver metastases: analysis of autopsy data. in Liver metastases; Weiss L, Gilbert HA, eds. Hall Medical Publishers Boston. 1982: 2-18.

3. Stern JS et al. Parallel Imaging in Hepatic Lesion Detection: Utilization of ASSET with Single-Shot Fast Spin Echo Imaging. Proc Intl. SOC Mag Reson Med. 2002: 10

4. Larsen $\mathrm{NE}$ et al. Quantitative liver ADC measurements using diffusion-weighted MRI at 3 Tesla: evaluation of reproducibility and perfusion dependence using different techniques for respiratory compensation. MAGMA. 2013 Oct;26(5):431-42.

5. Gourtsoyianni S, Papanikolaou N, Yarmenitis S, Maris T, Karantanas A, Gourtsoyiannis N. Respiratory gated diffusion-weighted imaging of the liver: value of apparent diffusion coefficient measurements in the differentiation between most commonly encountered benign and malignant focal liver lesions. Eur Radiol 2008;18:486-492.

6. Bruegel et al. Characterization of focal liver lesions by ADC measurements using a respiratory triggered diffusion-weighted single-shot echo-planar MR imaging technique. Eur Radiol 2008; 18: 477-485 DOI 10.1007/s00330-007-0785-9

7. Jones DK, Basser PJ. "Squashing peanuts and smashing pumpkins": how noise distorts diffusion-weighted MR data. Magn Reson Med 2004;52:979-993

8. Bitto et al. Optimum gradient factors for apparent diffusion coefficient measurements. Proc Intl Soc Mag Res Med 1995;913. 